01

\title{
Связанные солитонные состояния и локализация кноидальных волн на границе раздела нелинейной и линейной сред
}

\author{
(C) C.E. Савотченко \\ Белгородский государственный технологический университет им. В.Г. Шухова, \\ 308012 Белгород, Россия \\ e-mail: savotchenkose@mail.ru)
}

(Поступило в Редакцию 4 апреля 2017 г.)

Рассмотрены контактные явления на границе линейной и нелинейной сред. Решены вопросы существования различных видов стационарных состояний в двухуровневой системе с отличающимися параметрами закона дисперсии. На основе модели, использующей нелинейное уравнение Шредингера, показано, что в рассматриваемой системе в зависимости от значения энергии возникают связанные состояния солитонов, локализация волн по одну сторону от дефекта, трансформация нелинейной волны в линейную при переходе через границу раздела сред. Получены дисперсионные соотношения, определяющие значения энергии таких состояний.

DOI: $10.21883 /$ JTF.2017.12.45197.2282

\section{Введение}

Явления, происходящие вблизи контакта двух сред с различными физическими свойствами, играют важную роль в различных технических приложениях квантовой твердотельной электроники и оптоэлектроники. Особое значение в этом направлении имеет изучение характеристик прозрачности границы раздела, с чем связаны эффекты локализации элементарных возбуждений в кристаллах[1]. Новые особенности возникают в ангармонических кристаллах с дефектами, в которых отмечено существование локальных колебательных состояний с частотами вне зоны сплошного спектра, зависящими от амплитуды колебаний [2]. К настоящему времени существует большое количество теоретических работ, посвященных описанию малоамплитудных нелинейных колебаний, локализованных вблизи дефектов, использующих при формулировке математических моделей различные нелинейные уравнения, в том числе и нелинейное уравнение Шредингера (НУШ) [3].

НУШ широко применяется для формулировки моделей при исследовании полей различной физической природы: упругого, электрического и магнитного [4]. В частности, НУШ использовалось при описании эффектов локализации электромагнитных волн вблизи границ раздела нелинейных сред [5], где указано существование нелинейных локализованных возбуждений с несимметричным профилем, отличающихся от свободно распространяющихся солитонов, и называемых нелинейными поверхностными волнами. Предлагалось также и обобщение НУШ для среды с пространственной дисперсией [6].

Поэтому для описания новых эффектов, связанных с локализацией возбуждений различной физической природы вблизи дефектов, имеет смысл далее рассматривать математическую модель, использующую НУШ, которому подчиняется функция $\psi$, выступающая в роли огибающей комплексного поля компонент вектора намагниченности в легкоосном ферромагнетике, либо комплексной амплитуды упругого поля смещения сдвиговой волны в кубическом кристалле с плоским дефектом, либо комплексной функции из амплитуд компонент электрического поля в оптической нелинейной среде [3]. Тогда параметры в уравнениях будут иметь соответствующий физических смысл в рамках одной из трех указанных моделей.

При наличии плоского дефекта в виде границы раздела нелинейных сред с различными характеристиками НУШ использовалось в [7], где была рассмотрена проблема решения НУШ с модифицированным потенциалом с $\delta$-функцией, моделирующим взаимодействие возбуждения с точечным дефектом, обладающего внутренней структурой, в нелинейной среде при учете дальнодействующих сил межатомного взаимодействия.

В настоящей работе получены решения НУШ и определены энергетические уровни стационарных колебательных состояний вблизи дефекта в рамках модели, которая представляет собой обобщение двухуровневой системы, рассмотренной в $[8,9]$, для случая границы раздела нелинейной и линейной сред.

Наличие двух ветвей возбуждений с различающимися параметрами законов дисперсии и взаимодействие возбуждений с плоскостью контакта сред с различными характеристиками, в том числе и ангармонизмом, приводит к появлению целого набора стационарных состояний различных типов. Волновые функции в таких состояниях в зависимости от энергии возбуждений в полупространстве с линейной средой могут быть монотонно затухающими при удалении от границы раздела, или гармоническими колебаниями, а в полупространстве с нелинейной средой они могут иметь форму солитонов НУШ или кноидальных волн, соответствующих периодическим решениям НУШ. При формулировке математической модели взаимодействие ветвей возбуждений 
на границе раздела сред учитывается специфическими граничными условиями [8]. В результате комбинации различных типов решений линейного уравнения Шредингера (УШ) и НУШ, удовлетворяющих сформулированным граничным условиям, и возникает многообразие различных типов стационарных состояний, описывающих процессы трансформации и локализации волн при переходе через границу раздела сред.

\section{1. Формулировка математической модели}

Будем считать, что две среды с различными физическими характеристиками, в том числе и по критерию нелинейности, разделены плоской границей раздела, проходящей через начало координат, перпендикулярно оси $O x$. Полупространство в области $x<0$ занимает среда с гармоническим межатомным взаимодействием (линейная среда), а полупространство в области $x>0$ занимает среда с ангармоническим межатомным взаимодействием (нелинейная среда). Также предполагается, что возмущение параметров сред, создаваемое границей раздела как плоским дефектом, сосредоточено на расстояниях, существенно меньших размеров возбуждений, и в этом смысле оно может считаться локальным.

Рассмотрим взаимодействие линейных и нелинейных возбуждений, взаимодействующих вблизи дефекта на основе одномерной модели двухуровневой системы, в которой возбуждение на границе раздела сред, как плоском дефекте, может находиться в двух состояниях с различными энергиями $\Omega_{1}^{(+)}$и $\Omega_{2}^{(+)}$. Знак „, “ здесь и далее означает принадлежность параметра к характеристикам полупространства с нелинейной средой, а , - “ - линейной.

Вследствие локальности возмущения параметров сред взаимодействие границы раздела с возбуждением можно описывать короткодействующим потенциалом с $\delta$-функцией. Полная волновая функция двухуровневой системы с энергией $E$, подчиняющаяся стационарному уравнению Шредингера $H \Psi=E \Psi$, состоит из двух слагаемых, для которых возбуждение может находиться в первом или во втором состояниях (уровнях): $\Psi(x)=a_{1}^{+}|0\rangle \psi_{1}(x)+a_{2}^{+}|0\rangle \psi_{2}(x)$, где $a_{1,2}^{+}, a_{1,2}$ - операторы рождения и уничтожения возбуждения в первом и втором состояниях [9], а волновые функции $\psi_{1}(x)$ и $\psi_{2}(x)$ этих состояний (ветвей спектра) являются независимыми решениями линейного УШ в полупространстве с линейной средой и НУШ в полупространстве с нелинейной средой

$$
\begin{aligned}
E \psi_{j}(x)= & -\frac{1}{2 m_{j}} \psi_{j}^{\prime \prime}(x)+\Omega_{j}(x) \psi_{j}(x) \\
& -\gamma_{j} \theta(x)\left|\psi_{j}(x)\right|^{2} \psi_{j}(x)+U_{j}(x) .
\end{aligned}
$$

Здесь и далее индекс принимает два значения: $j=1,2$, использована функция Хевисайда

$$
\theta(x)= \begin{cases}0, & x<0, \\ 1, & x>0,\end{cases}
$$

$m_{j}$ - эффективная масса возбуждений, $\gamma_{j}>0-$ параметры нелинейности среды, расположенной справа от дефекта, $\delta(x)-\delta$-функция Дирака,

$$
\Omega_{j}(x)= \begin{cases}\Omega_{j}^{(-)}, & x<0, \\ \Omega_{j}^{(+)}, & x>0,\end{cases}
$$

$\Omega_{j}^{( \pm)}$- значения уровней дна энергетической зоны, $U_{j}$ - потенциалы, описывающие взаимодействие волн на границе раздела двух сред

$$
U_{1}(x)=\left(\alpha_{1} \psi_{1}+\beta \psi_{2}\right) \delta(x), \quad U_{2}=\left(\alpha_{2} \psi_{2}+\beta \psi_{1}\right) \delta(x),
$$

$\alpha_{j}, \beta$ - параметры интенсивности взаимодействия возбуждений на границе раздела сред.

Стационарные состояния с энергией $E$ уравнений (1) представимы в виде $\psi_{j}(x, t)=\psi_{j}(x) \exp (-i E t)$. Тогда решение уравнений (1) с потенциалами (2) сводится к решению стационарных УШ и НУШ:

$$
\begin{aligned}
\psi_{j}^{\prime \prime}(x) & +2 m_{j}\left(E-\Omega_{j}(x)\right) \psi_{j}(x) \\
& +2 m_{j} \gamma_{j} \theta(x)\left|\psi_{j}(x)\right|^{2} \psi_{j}(x)=0
\end{aligned}
$$

с граничными условиями

$$
\begin{gathered}
\psi_{j}(+0)=\psi_{j}(-0)=\psi_{j}(0), \\
\left\{\begin{array}{c}
\psi_{1}^{\prime}(+0)-\psi_{1}^{\prime}(-0)=2 m_{1}\left\{\alpha_{1} \psi_{1}(0)+\beta \psi_{2}(0)\right\}, \\
\psi_{2}^{\prime}(+0)-\psi_{2}^{\prime}(-0)=2 m_{2}\left\{\alpha_{2} \psi_{2}(0)+\beta \psi_{1}(0)\right\} .
\end{array}\right.
\end{gathered}
$$

В линейной среде без дефекта распространяются свободные волны с квадратичным законом дисперсии. При наличии дефекта в линейной среде возможно существование локализованных состояний с несимметричной частью волны, распространяющейся только по одну сторону от дефекта [8].

Решения НУШ могут быть солитонные и в виде кноидальных волн, типы которых определяются значением энергии возбуждения и знаком параметра нелинейности [10]. Состояния с энергиями обеих ветвей спектра, лежащими ниже дна зоны сплошного спектра, будем далее называть локализованными, а такие, у которых энергия одной ветви лежит ниже дна зоны, а другой внутри зоны сплошного спектра - квазилокальными. Рассмотрим далее основные типы возникающих в данной системе состояний в зависимости от их энергии. Во всех этих случаях при наличии дефекта ветви состояний не являются независимыми, а взаимодействуют на границе раздела сред, поэтому могут называться связанными состояниями. Если в структуру волновой функции одной из ветвей в полупространстве с нелинейной средой входит солитонное решение НУШ, то соответствующее связанное состояние можно называть солитонным. 


\section{2. Локализованные состояния}

В области ниже дна зоны любой из ветвей спектра, когда энергия возбуждений находится в диапазоне $E<\min \left(\Omega_{1,2}^{( \pm)}\right)$, существуют несколько типов локализованных состояний. Уравнения (3) имеют решения, удовлетворяющие граничным условиям (4) и (5), в виде

$$
\psi_{j}(x)= \begin{cases}A_{j}^{(-)} e^{\kappa_{j} x}, & x<0, \\ \frac{A_{j}^{(+)}}{\operatorname{ch} q_{j}\left(x-x_{j}\right)}, & x>0,\end{cases}
$$

где декременты затухания $\kappa_{j}^{2}=2 m_{j}\left(\Omega_{j}^{(-)}-E\right)$, $q_{j}^{2}=m_{j}\left(\Omega_{j}^{(+)}-E\right) ; \quad$ амплитуды $A_{j}^{(-)}=q_{j}\left(m \gamma_{j}\right)^{-1 / 2} / \operatorname{ch} a_{j} x_{j}, \quad A_{j}^{(+)}=q_{j}\left(m_{j} \gamma_{j}\right)^{-1 / 2}, \quad$ a уровни энергии определяются из дисперсионного соотношения

$$
\Delta_{t 1} \Delta_{t 2}=4 m_{1} m_{2} \beta^{2},
$$

где $\Delta_{t 1}=q_{j}$ th $q_{j} x_{j}-\kappa_{j}-2 m_{j} \alpha_{j}$.

В частном случае, когда $\alpha_{j}=\alpha>0, m_{j}=m$, $\Omega_{j}^{( \pm)}=\Omega$, получается, что $\kappa_{j}=q_{j}=q$. Тогда при условии, что $x_{1}=x_{2}=x_{0}$, из (7) вытекает упрощенное дисперсионное соотношение $q\left(\right.$ th $\left.q x_{0}-1\right)=2 m(\alpha \pm \beta)$. В предельном случае при $q x_{0} \ll 1$ из него выражается энергия локализованного состояния в явном виде

$$
E=\Omega-\frac{1}{8 m x_{0}^{2}}\left\{1 \pm\left[1+8 m x_{0}(\alpha \pm \beta)\right]^{1 / 2}\right\}^{2} .
$$

Состояние, описываемое волновой функцией (6), характеризует связанное состояние солитонов, локализованных несимметрично относительно границы раздела сред. Параметр $x_{0}$ является свободным, поэтому решение данного типа является однопараметрическим.

В рассматриваемом энергетическом диапазоне существует состояние, описываемое волновой функцией вида

$$
\psi_{j}(x)= \begin{cases}A_{d j}^{(-)} e^{\kappa_{j} x}, & x<0, \\ A_{d j}^{(+)} \mathrm{dn}\left(q_{d j}\left(x-x_{j}\right), k\right), & x>0,\end{cases}
$$

где $k$ - модуль эллиптической функции $\mathrm{dn}$ $(0<k<1)$, амплитуды $A_{d j}^{(-)}=\operatorname{dn}\left(q_{d j} x_{j}, k\right) /\left(m_{j} \gamma_{j}\right)^{1 / 2}$, $A_{d j}^{(+)}=q_{d j}\left(m_{j} \gamma_{j}\right)^{-1 / 2}, \quad$ декременты затухания $\kappa_{j}$ такие же как и для (6), волновое число $q_{d j}^{2}=2 m_{j}\left(\Omega^{(+)}-E\right) /\left(2-k^{2}\right), \quad$ а уровни энергии определяются из дисперсионного соотношения

$$
\Delta_{d 1} \Delta_{d 2}=4 m_{1} m_{2} \beta^{2},
$$

где $\Delta_{d j}=k^{2} q_{d j} \operatorname{sn}\left(q_{d j} x_{j}, k\right) \operatorname{sn}\left(q_{d j} x_{j}+K(k), k\right)-\kappa_{j}-2 m_{j} \alpha_{j}$, sn - эллиптический синус, $K(k)$ - полный эллиптический интеграл первого рода [10].

В частном случае, когда $\alpha_{j}=\alpha>0, m_{j}=m, \Omega_{j}^{( \pm)}=\Omega$, получается, что $\kappa_{j}=\kappa, q_{d j}=q_{d}, \kappa^{2}=q_{d j}^{2}\left(2-k^{2}\right)$. Тогда при условии, что $x_{1}=x_{2}=x_{0}$, из (10) получается дисперсионное соотношение

$$
\begin{gathered}
q_{d}\left(k^{2} \operatorname{sn}\left(q_{d} x_{0}, k\right) \operatorname{sn}\left(q_{d} x_{0}+K(k), k\right)-\left(2-k^{2}\right)^{1 / 2}\right) \\
=2 m(\alpha \pm \beta)
\end{gathered}
$$

В предельном случае при $q x_{0} \ll 1$ из (11) выражается энергия в явном виде

$$
\begin{aligned}
& E=\Omega-\frac{\left(2-k^{2}\right)^{2}}{8 m k^{4} x_{0}^{2}} \\
& \times\left\{1 \pm\left[1+8 m k^{2} x_{0}(\alpha \pm \beta) /\left(2-k^{2}\right)\right]^{1 / 2}\right\}^{2},
\end{aligned}
$$

Параметры $x_{0}$ и $k$ являются свободными, поэтому решение данного типа является двухпараметрическим.

В рассматриваемом диапазоне энергий существует решение НУШ (3) другого типа

$$
\psi_{j}(x)= \begin{cases}A_{c j}^{(-)} e^{\kappa_{j} x}, & x<0, \\ A_{c j}^{(+)} \operatorname{cn}\left(q_{c j}\left(x-x_{j}\right), k\right), & x>0,\end{cases}
$$

где cn - эллиптический косинус; амплитуды $A_{c j}^{(-)}=k q_{c j} /\left(m_{j} \gamma_{j}\right)^{1 / 2} \operatorname{cn}\left(q_{c j} x_{j}, k\right), A_{c j}^{(+)}=k q_{c j}\left(m_{j} \gamma_{j}\right)^{-1 / 2}$, $q_{c j}^{2}=2 m_{j}\left(\Omega^{(+)}-E\right) /\left(2 k^{2}-1\right)$, а уровни энергии определяются из дисперсионного соотношения

$$
\Delta_{c 1} \Delta_{c 2}=4 m_{1} m_{2} \beta^{2},
$$

где $\Delta_{c 1}=q_{c j} \operatorname{sn}\left(q_{c j} x_{j}, k\right) / \operatorname{sn}\left(q_{c j} x_{j}+K(k), k\right)-\kappa_{j}-2 m_{j} \alpha_{j}$.

В частном случае, когда $\alpha_{j}=\alpha>0, m_{j}=m, \Omega_{j}=\Omega$, получается, что $\kappa_{j}=\kappa, q_{c j}=q c, \kappa^{2}=q_{c j}^{2}\left(2 k^{2}-1\right)$. Тогда при условии, что $x 1=x 2=x_{0}$, из (14) получается дисперсионное соотношение

$$
\begin{gathered}
q_{c}\left(\operatorname{sn}\left(q_{c} x_{0}, k\right) / \operatorname{sn}\left(q_{c} x_{0}+K(k), k\right)-\left(2 k^{2}-1\right)^{1 / 2}\right) \\
=2 m(\alpha \pm \beta) .
\end{gathered}
$$

В предельном случае при $q x_{0} \ll 1$ из (15) выражается энергия в явном виде

$$
\begin{aligned}
E= & \Omega-\frac{\left(2 k^{2}-1\right)^{2}}{8 m x_{0}^{2}} \\
& \times\left\{1 \pm\left[1+8 m x_{0}(\alpha \pm \beta) /\left(2 k^{2}-1\right)\right]^{1 / 2}\right\}^{2} .
\end{aligned}
$$

Волновые функции (9) и (13) описывают локализацию кноидальных волн при переходе через границу раздела нелинейной и линейной сред. Относительно двух ветвей такие состояния можно назвать симметричными, так как обеим ветвям в полупространстве с нелинейной средой соответствуют кноидальные волны.

Помимо таких состояний в рассматриваемом диапазоне энергий могут реализовываться такие, в которых одной ветви соответствует кноидальная волна, а другой 
солитон. Существует решение уравнения (3), удовлетворяющее условием (3) и (4), описываемое волновыми функциями вида

$$
\begin{gathered}
\psi_{1}(x)= \begin{cases}A_{1}^{(-)} e^{\kappa_{1} x}, & x<0, \\
\frac{A_{1}^{(+)}}{\operatorname{ch} q_{1}\left(x-x_{1}\right)}, & x>0,\end{cases} \\
\psi_{2}(x)= \begin{cases}A_{d 2}^{(-)} e^{\kappa_{2} x}, & x<0, \\
A_{d 2}^{(+)} \operatorname{dn}\left(q_{d 2}\left(x-x_{2}\right), k\right), & x>0,\end{cases}
\end{gathered}
$$

в котором величины $\kappa_{j}$ и $q_{1}$, амплитуды $A_{1}^{( \pm)}$такие же как и для (6), $q_{d 2}$ и $A_{d 2}^{( \pm)}-$как и для (9), энергия определяется из дисперсионного соотношения

$$
\Delta_{t 1} \Delta_{d 2}=4 m_{1} m_{2} \beta^{2} .
$$

Кроме того, существует другое решение уравнения (3), удовлетворяющее условием (3) и (4), в котором волновая функция $\psi_{1}$ такая же, как и в (17), а $\psi_{2}$ имеет вид

$$
\psi_{2}(x)= \begin{cases}A_{c 2}^{(-)} e^{\kappa_{2} x}, & x<0 \\ A_{c 2}^{(+)} \operatorname{cn}\left(q_{c 2}\left(x-x_{2}\right), k\right), & x>0\end{cases}
$$

в котором величины $\kappa_{j}$ и $q_{1}$, амплитуды $A_{1}^{( \pm)}$такие же как и в предыдущем случае, а $q_{c 2}$ и $A_{c 2}^{( \pm)}-$как и для (13), энергия определяется из дисперсионного соотношения

$$
\Delta_{t 1} \Delta_{c 2}=4 m_{1} m_{2} \beta^{2} .
$$

Состояния вида (17) и (19) описывают локализацию нелинейной волны одной ветви с энергией в спектре локальных состояний другой ветви с несимметричным профилем относительно границы раздела сред.

\section{3. Трансформация и локализация волн в спектре квазилокальных состояний}

В области выше границы первой зоны, но ниже границы зоны второй ветви спектра, когда энергия возбуждений находится в диапазоне $\Omega_{1}^{(-)}<E<\min \left\{\Omega_{2}^{(-)}, \Omega_{2}^{( \pm)}\right\}$, существуют несколько типов квазилокальных состояний. К одному из таких типов относится состояние, описываемое решением уравнений (3), удовлетворяющим граничным условиям (4) и (5), в котором волновая функция второй ветви $\psi_{2}$ такая же, как и в $(6)$, а $\psi_{1}$ имеет вид

$$
\psi_{1}(x)= \begin{cases}A_{\varphi 1}^{(-)} \cos \left(p_{1} x+\varphi\right), & x<0, \\ \frac{A_{1}^{(+)}}{\operatorname{ch} q_{1}\left(x-x_{1}\right)}, & x>0,\end{cases}
$$

где $\quad p_{1}^{2}=2 m_{1}\left(E-\Omega_{1}^{(-)}\right), \quad A_{\varphi 1}^{(-)}=q_{1}\left(m_{1} \gamma_{1}\right)^{-1 / 2} / \cos \varphi$, остальные величины были определены выше, а энергия определяется из дисперсионного соотношения

$$
\Delta_{t 1}^{\varphi} \Delta_{t 2}=4 m_{1} m_{2} \beta^{2},
$$

где $\Delta_{t 1}^{\varphi}=q_{1}$ th $q_{1} x_{1}+p_{1} \operatorname{tg} \varphi-2 m_{1} \alpha_{1}$.

В частном случае, когда $\alpha_{j}=\alpha>0, m_{j}=m$, $\Omega_{2}^{( \pm)}=\Omega_{1}^{(+)} \neq \Omega_{1}^{(-)}, \quad$ из $\quad(22)$ получается, что $\kappa_{j}=q_{j}=q$. Тогда при условии, что $x_{1}=x_{2}=0$, из (22) получается дисперсионное соотношение:

$$
\operatorname{tg} \varphi=2 m\left\{2 m\left(\alpha^{2}-\beta^{2}\right)+\alpha q_{1}\right\} / p_{1}\left(2 m \alpha+q_{1}\right) .
$$

Состояние, описываемое волновой функцией (21), характеризует локализацию бегущей волны первой ветви при наличии связанного состояния второй ветви, локализованного несимметрично относительно границы раздела сред.

В рассматриваемом энергетическом диапазоне существует состояние, в котором для первой ветви волновая функция имеет вид

$$
\psi_{1}(x)= \begin{cases}A_{\varphi d 1}^{(-)} \cos \left(p_{1} x+\varphi\right), & x<0, \\ A_{d 1}^{(+)} \operatorname{dn}\left(q_{d 1}\left(x-x_{1}\right), k\right), & x>0,\end{cases}
$$

где параметры нелинейной волны в положительном полупространстве такие же, как и для (9), волновое число такое же, как и для (21), амплитуда $A_{\varphi d 1}^{(-)}=q_{d 1} /\left(m_{1} \gamma_{1}\right)^{1 / 2} \operatorname{dn}\left(q_{d 1} x_{1}, k\right) / \cos \varphi$. Волновая функция второй ветви $\psi_{2}$ такая же, как и в (9), а энергия такого состояния определяется из дисперсионного соотношения

$$
\Delta_{d 1}^{\varphi} \Delta_{d 2}=4 m_{1} m_{2} \beta^{2},
$$

где $\Delta_{d 1}^{\varphi}=k^{2} q_{d 1} \operatorname{sn}\left(q_{d 1} x_{1}, k\right) \operatorname{sn}\left(q_{d 1} x_{1}+K(k), k\right)+p_{1} \operatorname{tg} \varphi-$ $-2 m_{1} \alpha_{1}$.

В спектре квазилокальных состояний существует еще одно состояние, в котором для первой ветви волновая функция имеет вид

$$
\psi_{1}(x)= \begin{cases}A_{\varphi c 1}^{(-1)} \cos \left(p_{1} x+\varphi\right), & x<0, \\ A_{c 1}^{(+)} \operatorname{cn}\left(q_{c 1}\left(x-x_{1}\right), k\right), & x>0,\end{cases}
$$

где параметры нелинейной волны в положительном полупространстве такие же, как и для (13), волновое число такое же, как и для (21), амплитуда $A_{\varphi c 1}^{(-)}=q_{c 1} /\left(m_{1} \gamma_{1}\right)^{1 / 2} \operatorname{cn}\left(q_{c 1} x_{1}, k\right) / \cos \varphi$. Волновая функция второй ветви $\psi_{2}$ такая же, как и в (13), а энергия такого состояния определяется из дисперсионного соотношения

$$
\Delta_{c 1}^{\varphi} \Delta_{c 2}=4 m_{1} m_{2} \beta^{2},
$$

где $\Delta_{c 1}^{\varphi}=q_{c 1} \operatorname{sn}\left(q_{c 1} x_{1} k\right) / \operatorname{sn}\left(q_{c 1} x_{1}+K(k), k\right)+p_{1} \operatorname{tg} \varphi-$ $-2 m_{1} \alpha_{1}$.

Состояния вида (23) и (25) описывают трансформацию кноидальной волны в гармоническую для первой 
ветви и локализацию кноидальной волны для второй ветви при переходе через границу раздела сред. Другими словами, состояния спектра кноидальных волн в полупространстве с нелинейной средой при переходе через границу раздела сред попадают в спектр квазилокальных состояний в полупространстве с линейной средой.

Помимо таких состояний, в рассматриваемом энергетическом диапазоне могут реализовываться состояния, в которых волновая функция первой ветви $\psi_{1}$ такая же, как и (21), а второй ветви $\psi_{2}$ такая же, как и (9). Параметры решений были определены выше, а энергии таких состояний определяются из дисперсионного соотношения $\Delta_{t 1}^{\varphi} \Delta_{d 2}=4 m_{1} m_{2} \beta^{2}$. Также имеют место состояния для второго типа кноидальных волн, описываемые волновой функцией первой ветви $\psi_{1}$ такой же, как и (21), а второй ветви $\psi_{2}$ такой же, как и (13). Параметры решений были определены выше, а энергии таких состояний определяются из дисперсионного соотношения $\Delta_{t 1}^{\varphi} \Delta_{c 2}=4 m_{1} m_{2} \beta^{2}$.

Следует отметить, что также возможны еще два типа состояний. Для первого типа кноидальных волн (dn) волновая функция первой ветви $\psi_{1}$ такая же, как и (17), а второй ветви $\psi_{2}$ имеет форму вида (23). Для второго типа кноидальных волн (cn) волновая функция первой ветви $\psi_{1}$ остается той же формы (17), а второй ветви $\psi_{2}$ имеет форму вида (25). Дисперсионные соотношения для таких состояний будут соответственно представимы в виде $\Delta_{t 1} \Delta_{d 2}^{\varphi}=4 m_{1} m_{2} \beta^{2}$, где $\Delta_{d 2}^{\varphi}=$ $=k^{2} q_{d 2} \operatorname{sn}\left(q_{d 2} x_{2}, k\right) \operatorname{sn}\left(q_{d 2} x_{2}+K(k), k\right)+p_{2} \operatorname{tg} \varphi-2 m_{2} \alpha_{2}$ и $\quad \Delta_{t 1} \Delta_{c 2}^{\varphi}=4 m_{1} m_{2} \beta^{2}, \quad$ где $\quad \Delta_{c 2}^{\varphi}=q_{c 2} \operatorname{sn}\left(a_{c 2} x_{2}, k\right) /$ $\operatorname{sn}\left(q_{c 2} x_{2}+K(k), k\right)+p_{2} \operatorname{tg} \varphi-2 m_{2} \alpha_{2}$.

\section{4. Симметричные квазилокальные состояния}

В другой части спектра в области выше границы зоны спектра в полупространстве с линейной средой, но ниже границы зоны спектра в полупространстве с нелинейной средой, когда энергия возбуждений находится в диапазоне $\max \left\{\Omega_{1,2}^{(-)}\right\}<E<\min \left\{\Omega_{1,2}^{(+)}\right\}$, существуют несколько типов симметричных квазилокальных состояний. Под симметрией будем здесь понимать то, что волновые функции первой и второй ветвей имеют идентичный функциональный вид, а отличаются только значениями параметров.

К такому типу относится состояние, описываемое решением уравнений (3), удовлетворяющим граничным условиям (4) и (5), в котором волновые функции обеих ветвей имеют вид

$$
\psi_{j}(x)= \begin{cases}A_{\varphi j}^{(-)} \cos \left(p_{j} x+\varphi\right), & x<0, \\ \frac{A_{j}^{(+)}}{\operatorname{ch} q_{j}\left(x-x_{j}\right)}, & x>0,\end{cases}
$$

где $\quad p_{j}^{2}=2 m_{j}\left(E-\Omega_{j}^{(-)}\right), \quad A_{\varphi j}^{(-)}=q_{j}\left(m_{j} \gamma_{j}\right)^{-1 / 2} / \cos \varphi$, остальные величины были определены выше, а энергия определяется из дисперсионного соотношения

$$
\Delta_{t 1}^{\varphi} \Delta_{t 2}^{\varphi}=4 m_{1} m_{2} \beta^{2},
$$

где $\Delta_{t j}^{\varphi}=q_{k}$ th $q_{i} x_{j}+p_{j} \operatorname{tg} \varphi-2 m_{j} \alpha_{j}$.

В частном случае, когда $\alpha_{j}=\alpha>0, \quad m_{j}=m$, $\Omega_{j}^{(+)}=\Omega, \Omega_{j}^{(-)}=0$, получается, что $p_{j}=p=(2 m E)^{1 / 2}$, $q_{j}=q, \quad p^{2}=2 m \Omega-q^{2}$. Тогда при условии, что $x_{1}=x_{2}=x_{0}$, из (28) получается дисперсионное соотношение

$$
q \text { th } q x_{0}+p \operatorname{tg} \varphi=2 m(\alpha \pm \beta) .
$$

В предельном случае при $q x_{0} \ll 1$ из (29) энергия выражается в явном виде

$$
E=\Omega-\frac{\Omega \operatorname{tg}^{2} \varphi-2 m(\alpha \pm \beta)^{2}}{\operatorname{tg}^{2} \varphi-4 m x_{0}(\alpha \pm \beta)} .
$$

Состояние, описываемое волновыми функциями (27), характеризует локализацию волны при переходе через границу раздела из полупространства с линейной средой в полупространство с нелинейной средой.

В рассматриваемом диапазоне энергий существует решение НУШ (3) периодического типа

$$
\psi_{j}(x)= \begin{cases}A_{\varphi d j}^{(-)} \cos \left(p_{j} x+\varphi\right), & x<0, \\ A_{d j}^{(+)} \operatorname{dn}\left(q_{d j}\left(x-x_{j}\right), k\right), & x>0,\end{cases}
$$

где $A_{\varphi d j}^{(-)}=q_{d j} /\left(m_{j} \gamma_{j}\right)^{1 / 2} \operatorname{dn}\left(q_{d j} x_{j}, k\right) / \cos \varphi$, а остальные параметры определены ранее. Энергия такого состояния определяется из дисперсионного соотношения

$$
\Delta_{d 1}^{\varphi} \Delta_{d 2}^{\varphi}=4 m_{1} m_{2} \beta^{2} .
$$

В частном случае, когда $\alpha_{j}=\alpha>0, \quad m_{j}=m$, $\Omega_{j}^{(+)}, \quad \Omega_{j}^{(-)}=0, \quad$ получается, что $\quad p_{j}=p, \quad q_{d j}=q_{d}$ и $p^{2}=2 m \Omega-q_{d}^{2}\left(2-k^{2}\right)$. Тогда при условии, что $x_{1}=x_{2}=x_{0}$, из (32) получается дисперсионное соотношение

$k^{2} q_{d} \operatorname{sn}\left(q_{d} x_{0}, k\right) \operatorname{sn}\left(q_{d} x_{0}+K(k), k\right)+p \operatorname{tg} \varphi=2 m(\alpha \pm \beta)$.

В предельном случае при $q x_{0} \ll 1$ из (11) выражается энергия в явном виде

$$
E=\Omega-\left(2-k^{2}\right) \frac{\Omega \operatorname{tg}^{2} \varphi-2 m(\alpha \pm \beta)^{2}}{\left(2-k^{2}\right) \operatorname{tg}^{2} \varphi-4 k^{2} m x_{0}(\alpha \pm \beta)} .
$$

Для кноидальных волн второго типа в рассматриваемом диапазоне энергий волновые функции имеют вид

$$
\psi_{j}(x)= \begin{cases}A_{\varphi c j}^{(-)} \cos \left(p_{j} x+\varphi\right), & x<0 \\ A_{c j}^{(+)} \operatorname{cn}\left(q_{c j}\left(x-x_{j}\right), k\right), & x>0,\end{cases}
$$

где $A_{c j}^{(-)}=k q_{c j} /\left(m_{j} \gamma_{j}\right)^{1 / 2} \mathrm{cn}\left(q_{c j} x_{j}, k\right) / \cos \varphi$, а остальные параметры определены ранее. Энергия такого состояния определяется из дисперсионного соотношения

$$
\Delta_{c 1}^{\varphi} \Delta_{c 2}^{\varphi}=4 m_{1} m_{2} \beta^{2} .
$$


В частном случае, когда $\alpha_{j}=\alpha>0, m_{j}=m, \Omega_{j}^{( \pm)}=\Omega$, получается, что $p_{j}=p, q_{c j}=q_{c}$, . Тогда при условии, что $x_{1}=x_{2}=x_{0}$, из (36) получается дисперсионное соотношение

$$
q_{c} \operatorname{sn}\left(q_{c} x_{0}, k\right) / \operatorname{sn}\left(q_{c} x_{0}+K(k), k\right)+p \operatorname{tg} \varphi=2 m(\alpha \pm \beta) .
$$

В предельном случае при $q x_{0} \ll 1$ из (37) выражается энергия в явном виде

$$
E=\Omega-\left(2 k^{2}-1\right) \frac{\Omega \operatorname{tg}^{2} \varphi-2 m(\alpha \pm \beta)^{2}}{\left(2 k^{2}-1\right) \operatorname{tg}^{2} \varphi-4 m x_{0}(\alpha \pm \beta)} .
$$

Волновые функции (31) и (33) описывают трансформацию кноидальных волн в гармонические колебания при переходе через границу раздела нелинейной и линейной сред.

Рассмотренные квазилокальные состояния существуют не для всех значений параметра $\varphi$. Область допустимых его значений определяется соотношениями между характеристиками сред и дефекта.

\section{Заключение}

Следует отметить, что в настоящей работе были получены и классифицированы все возможные типы состояний для сред с положительным параметром нелинейности. Аналогичные типы состояний существуют и в средах с отрицательным параметром нелинейности, когда $\gamma=-g<0$. Как известно [10], при таком знаке нелинейности НУШ имеет всюду ограниченные решения в виде кинка $\psi(x)=A_{t}^{(+)}$th $q_{t}\left(x-x_{0}\right)$, где $A_{t}^{(+)}= \pm q_{t}(m g)^{-1 / 2}, \quad q_{t}^{2}=m(E-\Omega)$, а также периодическое решение, описываемое кноидальной волной $\psi(x)=A_{s}^{(+)} \operatorname{sn}\left(q_{s}\left(x-x_{0}\right), k\right), A_{s}^{(+)}= \pm q_{s}(m g)^{-1 / 2}$, $q_{s}^{2}=2 m(E-\Omega) /\left(1+k^{2}\right)$. Такие решения существуют при $E>\Omega$.

Для случая двухуровневой модели с двумя ветвями закона дисперсии состояния, описываемые при помощи таких видов решений, находятся в диапазоне энергий выше, чем аналогичные состояния в среде с положительной нелинейностью.

При $E<\Omega$ также НУШ имеет ограниченное решение в области $x>0$ в виде $\psi(x)=A_{\mathrm{sh}}^{(+)} / \operatorname{sh} q_{\mathrm{sh}}\left(x-x_{0}\right)$, $q_{\mathrm{sh}}^{2}=2 m(\Omega-E), A_{\mathrm{sh}}^{(+)}= \pm q_{\mathrm{sh}}(m g)^{-1 / 2}$, причем должно выполняться требование $x_{0}<0$. Данное обстоятельство обусловливает наличие более широкого набора состояний в двухуровневой системе на границе линейной и нелинейной сред. Однако по своей структуре все они будут аналогичны рассмотренным в настоящей работе типам состояний для среды с положительным ангармонизмом.

На основе модели двухуровневой системы, возбуждения в которой описываются НУШ, рассмотрены вопросы существования различных видов состояний в широком интервале энергетического спектра.
Использование полученных в настоящей работе результатов не ограничивается теорией ангармонических кристаллов, поскольку НУШ с различным знаком нелинейности, характеризующим притяжение или отталкивание, фокусировку и дефокусировку, находит широкое применение в теории магнитоупорядоченных сред, нелинейной оптике, динамике сверхтекучего жидкого гелия и в других системах.

\section{Список литературы}

[1] Kivshar Yu.S., Kosevich A.M., Chubykalo O.A. // Phys. Rev. A. 1990. Vol. 41. N 3. P. $1677-1688$.

[2] Богдан М.М., Герасимчук И.В., Ковалев А.С. // ФНТ. 1997. T. 23. № 2. C. 197-207.

[3] Герасимчук И.В., Ковалев А.С. // ФНТ. 2000. Т. 26. № 8. C. 799-809.

[4] Abdullaev F.Kh., Baizakov B.B., Umarov B.A. // Opt. Commun. 1998. Vol. 156. P. 341-346.

[5] Ахмедиев Н.Н., Корнеев В.И., Кузьменко Ю.В. // ЖЭТФ. 1985. T. 88. № 1. C. 107-115.

[6] Савотченко С.E. // Известия вузов. Физика. 2004. Т. 47. № 5. C. 79-84.

[7] Савотченко C.E. // Вестник Воронежского гос. ун-та. Сер.: Физика. Математика. 2016. № 4. С. 51-59.

[8] Косевич А.М. // ЖЭТФ. 1999. Т. 23. № 2. С. 197-207.

[9] Савотченко С.E. // Известия вузов. Физика. 2001. Т. 44. № 4. C. $67-73$.

[10] Давыдов А.С. Солитоны в молекулярных системах. Киев: Наукова думка, 1984. 288 с. 